Tohoku J. exp. Med., 1983, 141, Suppl., 687-692

\title{
Improved Gontrol in Self-Blood Glucose Monitoring Insulin Dependent Diabetics Treated with Acarbose
}

\author{
P. Zimmet, J. Wischusen, M. Cohen, L.R. Raper and C. \\ Crosbie \\ Diabetes Home Monitoring Centre and WHO Collaborating \\ Centre for the Epidemiology of Diabetes Mellitus, Royal \\ Southern Memorial Hospital, Melbourne, Australia
}

A major problem in the treatment of insulin dependent diabetes (IDDM) is the occurrence of post-prandial rises in blood glucose (BG) resulting in poor control of diabetes. Recently, there has been great interest in the use of dietary fiber to counter this problem ${ }^{1)}$. Fiber in the diet slows absorption of nutrients from the gastrointestinal tract, with the result that the post-prandial $B G$ response is flattened. Another approach has been the use of enzyme inhibitors such as the $\alpha$-glucosidase inhibitor acarbose ${ }^{2-7)}$. Acarbose is a competitive inhibitor of intestinal $\alpha$-glucosidases including sucrase, dextrinase, and maltase.

Acarbose slows the breakdown of starch and sucrose, and it has been shown to reduce the post-prandial rise in $\mathrm{BG}$ after carbohydrate test meals ${ }^{2,3)}$. More recently, Gérard and his co-workers have reported a reduction in post-prandial BG concentrations in IDDM subjects who were given acarbose over a two-month period $^{6)}$. Acarbose also significantly reduced mean daily BG and mean amplitude of glycemic excursions in these patients. Similar results were reported by Edmonds and his co-workers during a 4-week treatment period ${ }^{7}$.

The present study was designed to assess the effect of acarbose on the BG profile over a 2-week period in self-monitoring IDDM subjects.

\section{Patient and Methods}

Eleven IDDM subjects entered the study: 9 males and 2 females. Side effects were minimal and all subjects completed the study. The mean age and duration of diabetes in these patients are shown in Table 1.

In a double-blind crossover study, each subject received acarbose $100 \mathrm{mg}$ before each main meal for 2 weeks, and then after a two week washout period, placebo tablets for a further 2 weeks, or vice versa.

All subjects had been regularly self-monitoring their BG for at least 6 months prior to

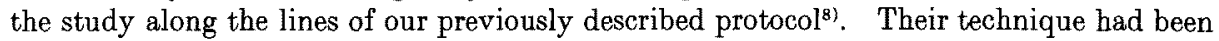


checked regularly by one of the authors (C.C.) In addition, during the study period, subjects were seen at 2-weekly intervals and the subject's own BG measurement was checked against the YSI glucose analyser. Each person maintained their regular diet and activity patterns.

At the beginning of the study and at the end of each treatment period, the following tests were performed-full blood count, serum electrolytes, creatinine, cholesterol, triglyceride, liver function tests, serum uric acid, calcium, and phosphate.

Blood glucose concentrations were measured using Dextrostix. and either a Dextrometer or Glucometer (Miles-Ames). On all 14 days of the study period, subjects measured BG before each main meal and supper. In addition, on 4 of the 14 days, BG measurements were performed 2 hours after each main meal. Some patients occasionally failed to perform a $B G$ measurement, and days where complete data were not available were excluded from the analysis.

Statistical analysis was performed using Student's $t$ test for paired data and, where appropriate, by analysis of variance.

The study was approved by the Royal Southern Memorial Hospital Ethics Committee, and all patients gave informed consent to participate.

As there was some variation (although not statistically significant) in mean fasting BG levels between the placebo and acarborse treatment periods, the results were expressed as $\Delta \mathrm{BG}$ (mmol/liter). This value is the change in $\mathrm{BG}$ from the fasting value to the respective post-breakfast, pre-and post-lunch, pre-and post-dinner and pre-supper value.

\section{Results}

\section{Mean pre-prandial blood glucose profiles}

The mean preprandial $\Delta \mathrm{BG}+$ s.E.M. (i.e. change in $\mathrm{BG}$ from fasting levelbefore lunch, dinner, and supper) for the placebo and acarbose periods is shown in

\section{DAILY PREPRANDIAL BLOOD GLUCOSE PROFILE}

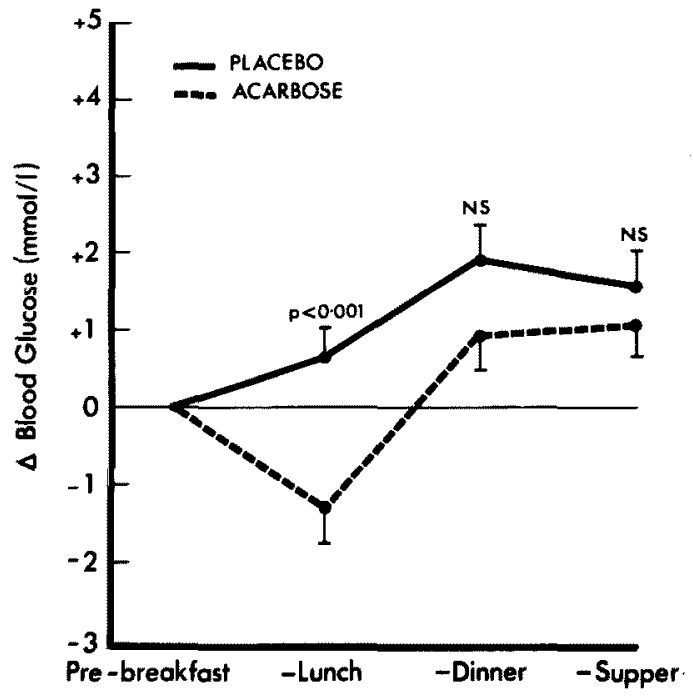

Fig. 1. Mean preprandial blood glucose profiles ( $\Delta \mathrm{BG} \pm$ s.E.M.) in the 11 IDDM patients. 


\section{DAILY PREPRANDIAL BLOOD GLUCOSE PROFILES}

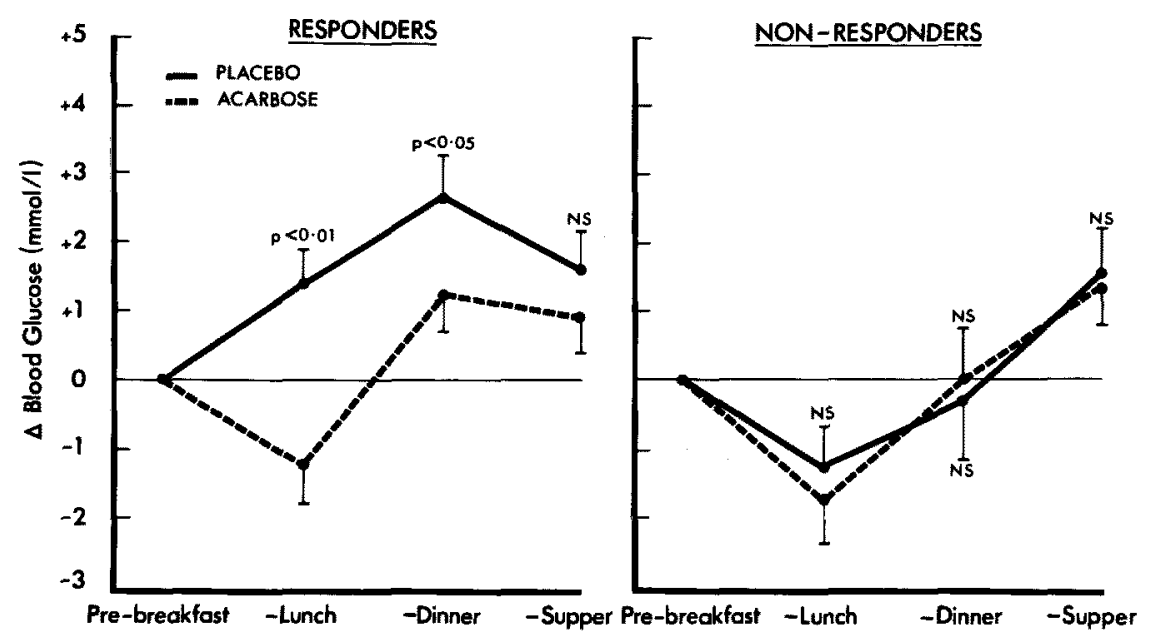

Fig. 2. Mean preprandial blood glucose profiles $(\Delta B G \pm$ S.E.M.) in acarbose responders and nonresponders.

Fig. 1. Preprandial BG levels during the day were consistently lower during acarbose therapy. The pre-lunch difference was highly significant $(p<0.001)$.

Analysis of individual patient data indicated that there were 8 responders and 3 non-responders to acarbose therapy. Non-response was defined as a difference in mean pre-lunch $\Delta \mathrm{BG}$ between placebo and acarbose treatment in an individual of $<1.0 \mathrm{mmol} /$ liter.

Fig. 2 shows daily mean $\Delta \mathrm{BG}$ profiles for placebo and acarbose periods in

\section{DAILY BLOOD GLUCOSE PROFILES - PRE AND POST-PRANDIAL}

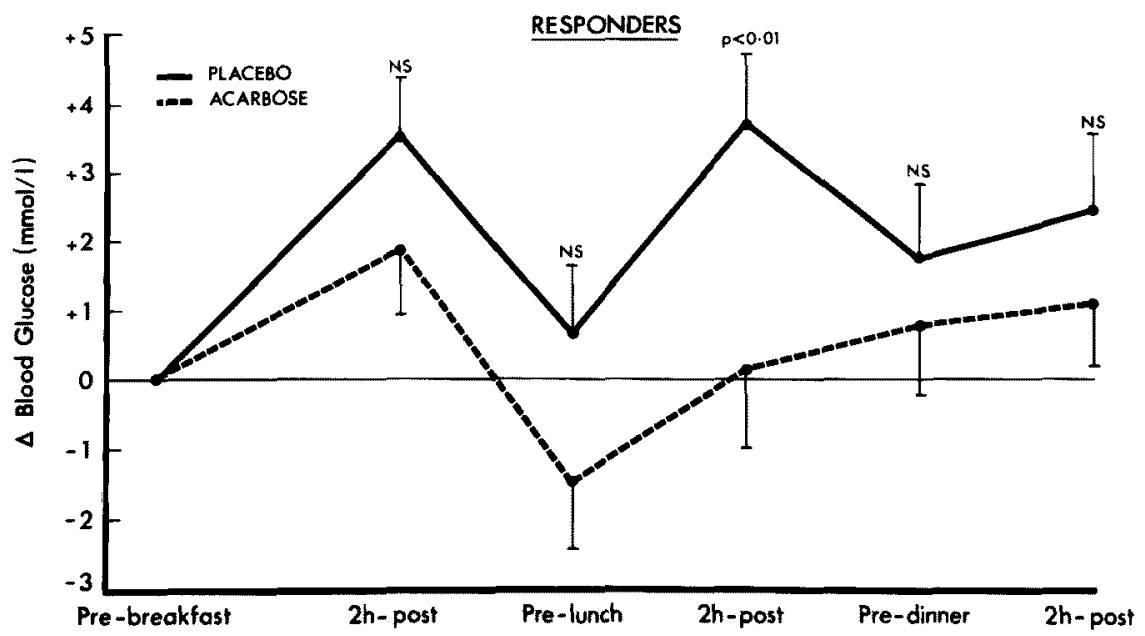

Fig. 3. Mean daily pre- and post-prandial blood glucose profiles $(\Delta B G \pm$ s.E.M.) in acarbose responders. 


\section{DAILY BLOOD GLUCOSE PROFILES - PRE AND POST - PRANDIAL}

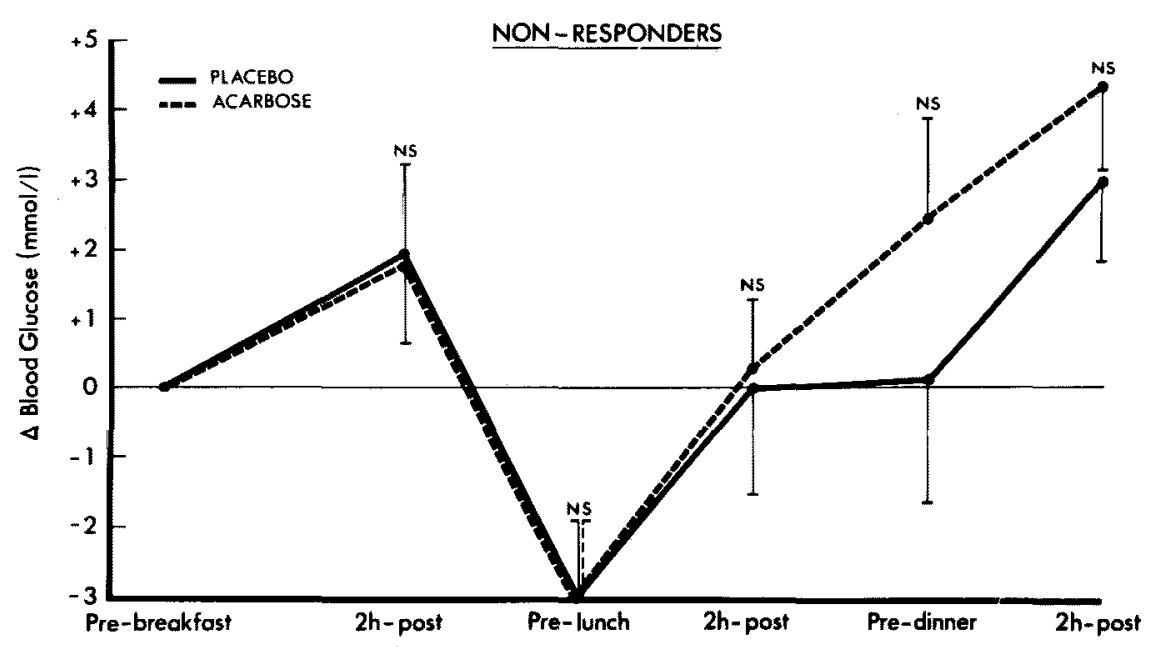

Fig. 4. Mean daily pre- and post-prandial blood glucose profiles $(\Delta B G \pm$ S.E.M $)$ in acarbose non-responders.

TABLE 1. Clinical characteristics of subjects studied*

\begin{tabular}{lcr}
\multicolumn{1}{c}{ Parameter } & Mean & Range \\
\hline Age (years) & 47.5 & $25-69$ \\
Duration diabetes (years) & 14.1 & $4-27$ \\
Insulin dose (units) & & \\
Placebo -before & 29.0 & $13-52$ \\
-after & 29.1 & $13-53$ \\
Acarbose-before & 29.5 & $13-53$ \\
-after & 29.2 & $13-53$ \\
\hline
\end{tabular}

* 11 patients ( 2 females and 9 males).

the responders and non-responders. In non-responders, BG profiles for placebo and acarbose were similar. However, in the responders, BG levels were lower throughout the day and the differences were significant-pre-lunch $(p<0.01)$ and pre-dinner $(p<0.05)$.

Mean daily pre-and post-prandial blood glucose profiles

On 4 of the 14 days, the subjects were asked to perform 2 hour post-prandial $\mathrm{BG}$ tests after each main meal, in addition to the preprandial tests. As some patients occasionally failed to perform a $\mathrm{B} G$ estimation, data analysis only included days where a full $\mathrm{BG}$ profile was done. The daily mean $\boldsymbol{\Delta} \mathrm{BG}$ profiles for placebo and acarbose for responders and non-responders (as defined earlier) are shown in Figs. 3 and 4 respectively. In the responders, BG levels were lower at all times during these days, although the difference between placebo and acarbose 
was only significant for the lunch post-prandial level $(p<0.01)$. In the nonresponders, $B G$ levels showed virtually no difference during the placebo and acarbose treatment periods.

There was no significant change in insulin dosage during either the placebo or acarbose treatment periods (Table 1).

\section{Discussion}

Many of the earlier reports on the use of acarbose in diabetes were performed in metabolic wards on inpatients, or on outpatients attending on specific days during the study $y^{2,4,5,6)}$. This study was designed to examine the effect of acarbose in self-monitoring IDDM subjects in the context of their normal lifestyle. Acarbose reduced the 2 hour post-prandial rise in $B G$, although this was only statistically significant after the midday meal. There was also a significant effect of acarbose in reducing the pre-lunch BG and, in most instances, mean BG levels during the day were lower during acarbose therapy. Similar results have been reported by Edmonds and co-workers in self-monitoring IDDM patients studied during 4 weeks of acarbose therapy ${ }^{7}$.

These workers also reported a reduction in total mean glucose, mean amplitude of glycemic excursions (MAGE) and the M-value during acarbose therapy ${ }^{7}$. Similar results were reported by Gerard and his co-workers in IDDM subjects receiving acarbose for two months ${ }^{6}$.

In this study, 3 subjects were classified as "non-responders" to acarbose. When these subjects were excluded from data analysis, the differences in mean pre-lunch and pre-dinner blood glucose valuse (acarbose versus placebo) were 2.6 and $1.5 \mathrm{mmol} /$ liter respectively.

The most disturbing side-effect of acarbose noted in other studies is flatulence ${ }^{2-7,9)}$. In this study, several patients reported mild flatulence but this side-effect never necessitated reduction of dosage or cessation of therapy. As the earlier reports using similar dosages are studies performed in Europe, it seems possible that subtle differences in the carbohydrate content of the diet between Australia and Europe may account for the milder side-effects in our subjects. On the other hand, this is only a small series of patients and the difference may be due to chance alone.

The results of this study suggest that through its effect in reducing postprandial rises in blood glucose, acarbose may prove to be a useful additional therapeutic agent for improving metabolic control in subjects with insulin-dependent diabetes mellitus.

Current available evidence favors the suggestion that the complications of diabetes are secondary to the metabolic disorder ${ }^{10)}$. Thus, the complications should be prevented, or at least reduced, by good control of the diabetic state. An important question is whether the beneficial effect of acarbose in improving metabolic control can be extended to reduction of complications in human 
diabetes. Of considerable interest then is the recent report by Lee of chronic treatment of genetically diabetic mice $(\mathrm{db} / \mathrm{db})$ with acarbose ${ }^{11}$. With improved metabolic control, there was a dose-dependent amelioration of diabetic nephropathy.

Longer term clinical studies are required to determine whether these findings can be extended to humans.

\section{Acknowledgments}

We are grateful for the most helpful advice of Dr M. Lawrie, Bayer Pharmaceutical Co., Australia, and Dr I. Hillebrand, Bayer AG, Germany in the planning of the study, and also for the supply of placebo and acarbose tablets. We thank Miss $R$. Swan for preparation of the manuscript and Mrs. A. Malpass for the art work.

\section{References}

1) Cudworth, A.G. (1979) Type 2 (Insulin-independent) diabetes. Diabetologia, 17, $67-69$.

2) Walton, R.J., Sherif, I.T., Noy, G.A. \& Alberti, K.G.M.M. (1979) Improved metabolic profiles in insulin-treated diabetic patients given an alpha-glucoside hydrolase inhiboitor. Brit. med. J., 1, 220-221.

3) Hillebrand, I., Boehme, K., Franck, G., Fink, H. \& Berchtold, P. (1979) The effects of the $\alpha$-glucosidase inhibitor BAY g 5421 (Acarbose) on meal stimulated elevation of circulating glucose, insulin and triglyceride levels in man. Res. exp. Med., 175, 8186.

4) Jenkins, D.J.A., Taylor, R.H., Nineham, R., et al. (1979) Combined use of guar and acarbose reduction of post-prandial glycaemia. Lancet, 2, 924-927.

5) Sachse, G. \& Willms, B. (1979) Effect of the $\alpha$-glucosidase-inhibitor BAY-g-5421 on blood glucose control of sulphonylurea-treated diabetics and insulin-treated diabetics. Diabetologia, 17, 287-290.

6) Gerard, J., Luyckx, A.S. \& Lefebvre, P.J. (1981) Improvement of metabolic control in insulin dependent diabetics treated with $\alpha$-glucosidase inhibitor acarbose for two months. Diabetologia, 21, 446-451.

7) Edmonds, M.E., Doddridge, M., John, P.N., White, J.M. \& Watkins, P.J. (1982) Acarbose (Bay g-54l) and post-prandial glycaemia in the home blood glucose profile. In: Genetic Enviromental Interaction in Diabetes Mellitus, edited by J.S. Melish, J. Hanna \& S. Baba, Excerpta Medica, Amsterdam, pp. 336-338.

8) Cohen, M. \& Zimmet, P. (1980) Home blood-glucose monitoring; a new approach to the management of diabetes mellitus. Med. J. Aust., 2, 713-716.

9) Taylor, R.H., Jenkins, D.J.A., Barker, H.M., Fielden, H., Goff, D.V., Misiewicz, J.J., Lee, D.A., Allen, H.B., MacDonald, G. \& Wallrabe, H. (1982) Effect of acarbose on the 24-hour blood glucose profile and pattern of carbohydrate absorption. Diabetes Care, 5, 92-96.

10) Skyler, J. (1979) Complications of diabetes mellitus: relationships to metabolic dysfunction. Diabetes Care 2, 499-509.

11) Lee, S.M. (1982) The effect of chronic $\alpha$-glucosidase inhibition on diabetic nephropathy in the $\mathrm{db} / \mathrm{db}$ mouse. Diabetes, 31, 249-254. 